\title{
Administración del sulfato ferroso y el incremento de hemoglobina en niños de nivel inicial con anemia en el Distrito de Huancayo 2016.
}

Administration of ferrous sulfate and the increase of hemoglobin in children with an initial level of anemia in the District of Huancayo 2016.

${ }^{1}$ Mercedes Acosta Román,${ }^{2}$ Jovavanna Luz Rojas Acosta ${ }^{a}$ y ${ }^{2}$ Elizabeth Jesús Poma ${ }^{a}$

\section{RESUMEN}

El OBJETIVO de estudio fue determinar la influencia de la administración del sulfato ferroso en los niveles de hemoglobina en niños de nivel inicial con anemia del ámbito del Programa Nacional de Alimentación Escolar Qali Warma del Distrito de Huancayo. METODOLOGIA: Investigación analítica, diseño ex post facto. La muestra estuvo conformada por 220 niños de nivel inicial de las 19 Instituciones Educativas estatales, se empleó el muestreo aleatorio al azar. La técnica utilizada fue la observación experimental con 2 instrumentos de hojas o fichas de registros de datos, que evaluó las siguientes variables: Administración del sulfato ferroso y el incremento de hemoglobina en niños con anemia. Los datos obtenidos a través de los cuestionarios fueron procesados con el programa Excel y SPSS. Los RESULTADOS indican que, la Influencia de la administración del sulfato ferroso a los niños de inicial del distrito de Huancayo esta categorizado en excelente, se llegó a la CONCLUSION que la administración del sulfato ferroso influye en el incremento de hemoglobina en niños de nivel inicial con anemia en el Distrito de Huancayo.

Palabras clave: Influencia, sulfato ferroso e incremento de hemoglobina.

\begin{abstract}
The OBJECTIVE of the study was to determine the influence of the administration of ferrous sulfate on hemoglobin levels in children of initial level with anemia of the National School Feeding Program Qali Warma of the District of Huancayo. METHODOLOGY: The type of analytical research, ex post facto design was used. The sample consisted of 220 children of the initial level of the 19 State Educational Institutions, random random sampling was used. The technique used was the experimental observation with 2 sheet instruments or records of data records, which evaluated the following variables: Administration of ferrous sulfate and the increase of hemoglobin in children with anemia. The data obtained through the questionnaires were processed with the Excel program and SPSS. The RESULTS indicate that, the Influence of the ferrous sulfate administration to the children of the district of Huancayo is categorized in excellent, CONCLUSION was reached that the administration of ferrous sulphate influences the increase of hemoglobin in children of initial level with anemia in the District of Huancayo.
\end{abstract}


keyword: Influence, ferrous sulfate and increase in hemoglobin.

${ }^{1}$ Universidad Nacional Autónoma de Tayacaja Daniel Hernández Morillo, ${ }^{2}$ Centro de Salud la Libertad - Huancayo.

${ }^{a}$ Licenciada en Enfermería. 


\section{INTRODUCCIÓN}

La nutrición es el proceso a través del cual el organismo absorbe y asimila las substancias necesarias para el funcionamiento del cuerpo. Este proceso biológico es uno de los más importantes determinantes para el óptimo funcionamiento y salud del cuerpo de los niños. Hasta perdurar incluso en la edad adulta; de ahí la importancia de brindar una orientación (Referencia)adecuada y oportuna a los docentes y familiares responsables de la atención y cuidado de los pre escolares, que ayudan a proteger y cuidar la salud. Este estudio tuvo como objetivo determinar la influencia de la administración del sulfato ferroso en los niveles de hemoglobina en niños con anemia de nivel inicial del ámbito del Programa Nacional de Alimentación Escolar Qali Warma del Distrito de Huancayo 2016.

El propósito de la investigación, tiene por finalidad diseñar nuevas estrategias destinadas a motivar y despertar el interés de la población pre escolar, buscando mantener los buenos hábitos alimenticios con alimentos ricos en hierro para prevenir la anemia en el pre escolar.

La importancia del estudio al conocer la influencia de la administración del tratamiento con sulfato ferroso en el incremento de los niveles de hemoglobina en niños con diagnóstico de anemia del nivel inicial, permitirá una mejor y oportuna intervención por parte de los profesionales y docentes de las instituciones educativas involucradas en el manejo de los niños con el diagnostico de anemia.

\section{MATERIAL Y MÉTODOS}

\section{ÁREADE ESTUDIO}

El presente estudio se ubica en el Distrito de Huancayo. El distrito de Huancayo es uno de los veintiocho que conforman la provincia de Huancayo, ubicada en el departamento de Junin. Tiene un área de 237,55 kilómetros cuadrados. Con una altitud media de $3249 \mathrm{~m}$ s. n.m.

\section{DESCRIPCIÓN DEL ÁREA DE ESTUDIO}

El presente estudio se realizó en las Instituciones Educativas estatales del ámbito Qali Warma del nivel inicial del Distrito de Huancayo del Departamento de Junín. Los cuales constan de 19 iniciales. Estas instituciones dirigidas por sus respectivos directores, con secciones de 3. 4. y 5 años, con una población escolar total de 2084 alumnos.

\section{METODOLOGÍA}

\section{TIPO DE INVESTIGACIÓN}

Teniendo en cuenta la naturaleza del problema y las variables en estudio, es una investigación de tipo descriptivo, porque describe el fenómeno de las variables sin ningún tipo de manipulación; de corte transversal retrospectivo porque se estudiará a las variables simultáneamente 


\section{POBLACIÓN Y MUESTRA}

La unidad de análisis para el estudio estuvo determinada por los escolares de nivel inicial de las 19 Instituciones Educativas estatales del ámbito Qali Warma del Distrito de Huancayo. La población total es de 517 alumnos de nivel inicial con diagnóstico de anemia.

Realizando las operaciones correspondientes el tamaño mínimo de la muestra es de 220 niños del nivel inicial de Huancayo, para un nivel de confianza del $95 \%$.

\section{TÉCNICAS E INSTRUMENTOS DE RECOLECCIÓN DE DATOS}

La técnica a emplear en la presente investigación fue la observación experimental con el instrumento la hoja o ficha de registro de datos.

Los 2 instrumentos pasaron por un juicio de expertos para su validez mediante la prueba $\mathrm{V}$ de Aiken, el instrumento sobre administración de sulfato ferroso obteniendo $(\mathrm{V}=0,92)$ y el instrumento de incremento de hemoglobina obteniendo $(\mathrm{V}=1,00)$. Por tanto, los dos instrumentos fueron válidos.

La prueba utilizada para comprobar la confiabilidad del instrumento fue el coeficiente del Alfa de Cronbach, para medir la consistencia interna de los ítems, obteniendo como resultado 0.77 siendo éste considerado según la escala de interpretación de la confiabilidad como Excelente lo que indica que el instrumento es confiable.
VARIABLE ADMINISTRACIÓN DEL SULFATO FERROSO.

El instrumento que se utilizó fue la hoja o ficha de registro de datos estructurado por las investigadoras, el cual tuvo por objetivo proporcionar información sobre la administración del sulfato ferroso a los niños con anemia del nivel inicial. Estuvo constituido por 2 preguntas cerradas con alternativas múltiples, el cual constó de las siguientes partes:

Parte I: Incluyó los datos generales de las personas que incluyen la muestra en estudio.

Parte II: Contenía los 2 ítems relacionados con el problema de estudio: Administración del sulfato ferroso según dosificación médica. Y Administración del sulfato ferroso respecto al consumo de los días de tratamiento. A cada respuesta se le asignó un puntaje de la siguiente forma:

\begin{tabular}{ll}
\hline \multicolumn{1}{c}{ ITEMS 1 } & \multicolumn{1}{c}{ ITEMS 2 } \\
\hline - No tomo la & - No tomo el \\
dosis (1 & tratamiento (1 \\
PUNTO) & PUNTO) \\
- Mayormente & - Dejo de tomar \\
rechaza la & un mes a más (2 \\
dosis (2 & PUNTOS) \\
PUNTOS) & - Dejo de tomar de \\
- A veces & 1 a 2 semanas (3 \\
rechaza la & PUNTOS) \\
dosis (3 & - Dejo de tomar \\
PUNTOS) & por menos de \\
- Pocas veces & una semana (4 \\
rechaza la & PUNTOS) \\
dosis (4 & - Siempre toma \\
PUNTOS) & todos los días de \\
- Siempre toma & clases (5 \\
toda la dosis (5 & PUNTOS) \\
PUNTOS) & \\
\hline
\end{tabular}

Obteniéndose así en todo el instrumento:

Puntaje máximo: 10 puntos.

Puntaje mínimo: 2 puntos. 
Para la categorización de la variable "administración de sulfato ferroso" se utilizó la Escala de Likert adaptada al número de preguntas del instrumento. Con lo que los resultados quedaron establecidos de la siguiente manera:

- Administración del sulfato ferroso Pésimo: 2-3

- Administración del sulfato ferroso Malo: 4-5

- Administración del sulfato ferroso Regular: 6-7

- Administración del sulfato ferroso Bueno: 8-9

- Administración del sulfato ferroso Excelente: 10

VARIABLE INCREMENTO DE HEMOGLOBINA EN NIÑOS CON ANEMIA.

El instrumento que se utilizó fue la hoja o ficha de registro de datos estructurado por las investigadoras, el cual tuvo por objetivo proporcionar información sobre el incremento de Hemoglobina en niños con anemia del nivel inicial. Estuvo constituido por 4 preguntas, 2 abiertas y 2 cerradas con alternativas múltiples, el cual constó de las siguientes partes:

Parte I: Incluyó los datos generales de las personas que incluyen la muestra en estudio.

Parte II: Contenía los 4 ítems relacionados con el problema de estudio.

Los ítems de las preguntas abiertas fueron para conocer el valor de hemoglobina antes y después de la administración del sulfato ferroso, pero los ítems de preguntas cerradas fueron para calificar los resultados antes y después, asignando un puntaje de la siguiente forma:

-NORMAL: 3 PUNTOS

-ANEMIALEVE: 2 PUNTOS

-ANEMIA MODERADA: 1 PUNTO

Obteniéndose así en todo el instrumento tomando en cuenta el resultado final de la hemoglobina:

- Puntaje máximo: 3 puntos.

- Puntaje mínimo: 1 punto.

Para la categorización de la variable "incremento de hemoglobina en niños con anemia" se utilizó la Escala ordinal adaptada a la pregunta final del instrumento. Con lo que los resultados quedaron establecidos de la siguiente manera:

- NORMAL: 3 PUNTOS

-ANEMIALEVE: 2 PUNTOS

-ANEMIA MODERADA: 1 PUNTO

\section{ANÁLISIS Y PROCESAMIENTO DE DATOS \\ E1 tratamiento estadístico de la información se realizó siguiendo el proceso siguiente:}

- Seriación: Se asignó un número de serie, correlativo a cada ficha de registro, lo que permitió tener un mejor tratamiento y control de los mismos.

- Codificación: Se elaboró un libro de 
códigos, donde se asigna un código a cada ítem de respuesta, con ello se logró un mayor control del trabajo de tabulación.

Se procedió al vaciamiento de los mismos en una matriz de datos electrónica, haciendo uso de la hoja de cálculo de Excel(Windows XP 2016), seguidamente se trasladaron los al Programa Estadístico SPSS versión 22.

- Tabulación: Mediante la aplicación de la técnica matemática de conteo, se realizó la tabulación extrayendo la información, ordenándola en cuadros simples y de doble entrada con indicadores de frecuencia y porcentaje.

- Graficación: Una vez tabulada la encuesta, se procedió a graficar los resultados en gráficas de barra.

Todo lo que sirvió para llevar los resultados al análisis e interpretación de los mismos.

Una vez obtenidos los datos, se procedió a analizar cada uno de ellos, atendiendo a los objetivos y variables de investigación; de manera tal que se pudo contrastar hipótesis con variables y objetivos, y así demostrar la validez o invalidez de estas.

Al final se formularán las conclusiones y recomendaciones para mejorar la problemática investigada.

\section{RESULTADOS}

TABLA N². Sexo de los niños con administración de sulfato ferroso de los iniciales del Distrito de Huancayo 2016

\begin{tabular}{|c|c|c|c|c|c|}
\hline $\mathrm{Se}$ & & 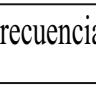 & & \multicolumn{2}{|c|}{ Porcentaje Porcentaje } \\
\hline (1000 & $\begin{array}{l}\text { Femenino } \\
\text { Masculin }\end{array}$ & $\begin{array}{ll}0 & 108 \\
\text { no } & 112\end{array}$ & $\begin{array}{l}49,1 \\
50,9\end{array}$ & & $\begin{array}{l}49,1 \\
100,0\end{array}$ \\
\hline & Total & 220 & 100,0 & 100,0 & \\
\hline
\end{tabular}

Fuente: Ficha de recolección de datos de historias clínicas aplicado por los investigadores.

El sexo de los niños en estudio es mayor el masculino con un $50.9 \%$ y el femenino con un $49.1 \%$.

Teniendo en cuenta que la ingesta de proteínas por día según gramos para las edades de 3 años es de $13 \mathrm{~g}$ y de 4 a 8 años es de $19 \mathrm{~g}$. Sin importar el sexo.

El que el porcentaje mayor fue de sexo masculino no es relevante para el estudio, respecto a su diagnóstico de tener anemia.

TABLA No ${ }^{\circ}$. Edad de los niños de nivel inicial con anemia del Distrito de Huancayo 2016

\begin{tabular}{|c|c|c|c|c|}
\hline Edad & Frecuencia & Porcentaje & $\begin{array}{l}\text { Porcentaje } \\
\text { válido }\end{array}$ & $\begin{array}{l}\text { Porcentaje } \\
\text { acumulado }\end{array}$ \\
\hline Válido3 & 22 & 10,0 & 10,0 & 10,0 \\
\hline 4 & 67 & 30,5 & 30,5 & 40,5 \\
\hline 5 & 105 & 47,7 & 47,7 & 88,2 \\
\hline 6 & 26 & 11,8 & 11,8 & 100,0 \\
\hline Tota & 220 & 100,0 & 100,0 & \\
\hline
\end{tabular}

Fuente: Ficha de recolección de datos de historias clínicas aplicado por los investigadores.

La edad de los niños con anemia de la muestra en estudio es mayor en las edades de 5 y 4 años con un $88.2 \%$ y $40.5 \%$ respectivamente. Lo cual indica que en estas dos edades tienen en su mayoría una alimentación deficiente en hierro. 
Figura $N^{0} 1$. Porcentaje del diagnóstico inicial de los niños de nivel inicial con anemia en el Distrito de Huancayo 2016

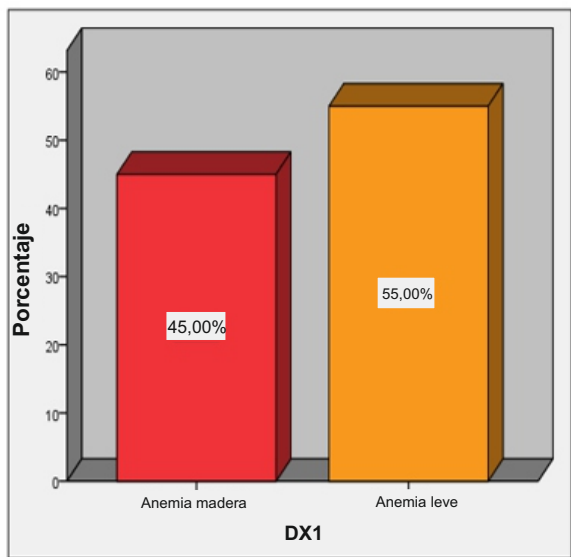

Fuente: Ficha de recolección de datos de historias clínicas aplicado por los investigadores.

En esta figura $\mathrm{N}^{\mathrm{o}} 1$ se observa que del total de los niños en estudio, que fueron 220, el diagnóstico inicial de la mayoría es anemia leve con un 55\%, seguido de un $45 \%$ con anemia moderada. Por que se debe tener en cuenta que el diagnóstico inicial de la anemia en estos niños no posee mucha diferencia porcentual.

Figura $\mathrm{N}^{\mathbf{2} 2}$. Administración del sulfato ferroso según la dosificación en los niños de nivel inicial con anemia en el Distrito de Huancayo 2016

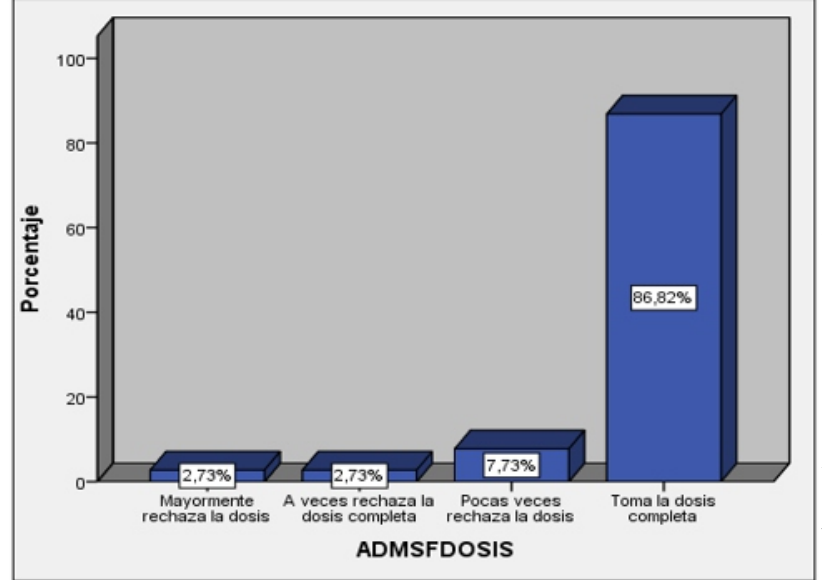

En esta figura $\mathrm{N}^{\circ} 3$ se puede observar que la aceptación de la administración del sulfato ferroso respecto al cumplimiento de la frecuencia del periodo del tratamiento por parte de los niños fue en un $86,36 \%$ que tomaron todos los días de
Fuente: Ficha de recolección de datos de historias clínicas aplicado por los investigadores.

Fuente: Ficha de recolección de datos de investigadores.

En esta figura $\mathrm{N}^{\circ} 2$ se puede observar que la aceptación de la administración del sulfato ferroso respecto al cumplimiento de la dosis que indico el médico a los niños fue en un $86,82 \%$ que tomaron la dosis completa, lo cual es lo correcto y favorece a su recuperación, seguido de un $7.73 \%$ que pocas veces rechaza la dosis, $2.73 \%$ a veces rechaza la dosis completa y un $2.73 \%$ mayormente rechaza la dosis.

FiguraN'3. Administración del sulfato ferroso según la frecuencia del consumo durante el periodo de tratamiento en los niños de nivel inicial con anemia en la en el Distrito de Huancayo 2016

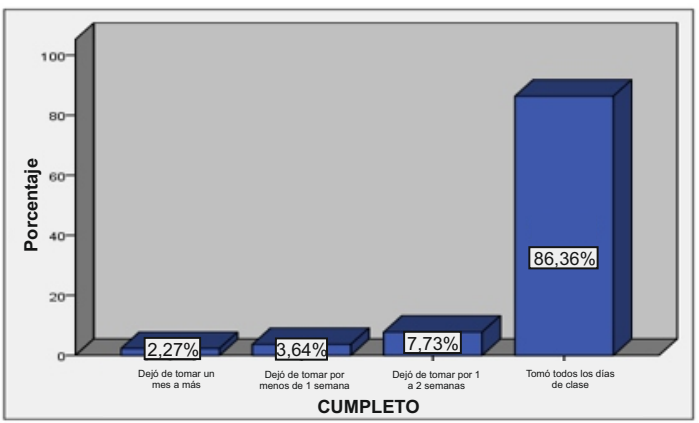
historias clínicas aplicado por los 
clases garantizando la continuidad del tratamiento durante sus días de clases, seguido de un $7.73 \%$ dejo de tomar por menos de 1 semana, 3.64\% dejo de tomar por 1 a 2 semanas y un $2.27 \%$ dejo de tomar un mes a más. Lo que se pude detallar es que los niños al estar estudiando de lunes a viernes y tomando en cuenta que la administración del sulfato ferroso fue por parte de las profesoras de nivel inicial. Esto se dio de lunes a viernes, durante un mes.

Figura $\mathrm{N}^{\circ} 4$. Resultado de la administración del sulfato ferroso a los niños de nivel inicial con anemia del Distrito de Huancayo 2016

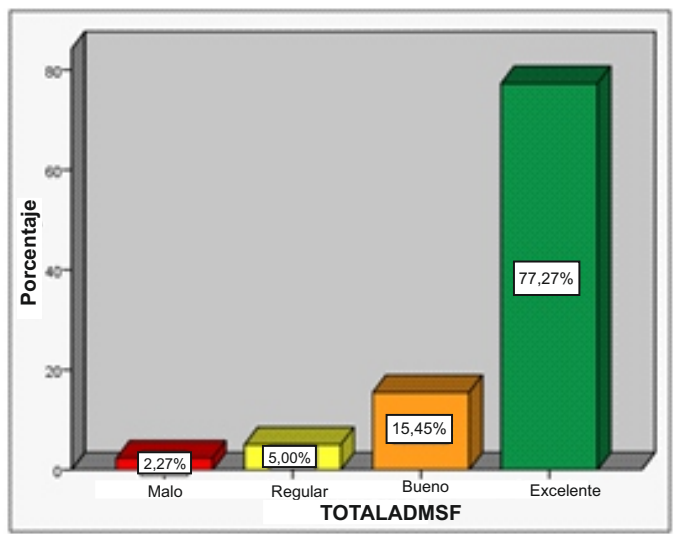

Fuente: Ficha de recolección de datos de historias clínicas aplicado por los investigadores.

Según la figura No4 la Influencia de la administración del sulfato ferroso a los niños de inicial del Distrito de Huancayo en general teniendo en cuenta la dosificación adecuada y el cumplimiento diario de la administración esta categorizado en excelente en un porcentaje de $76.29 \%$, en bueno en un
$15.81 \%$, en regular $5.15 \%$ y en malo en un $2.75 \%$.

Figura $\mathrm{N}^{0} 5$. Porcentaje del diagnóstico final después de la administración del sulfato ferroso en los niños de nivel inicial con anemia en el Distrito de Huancayo 2016

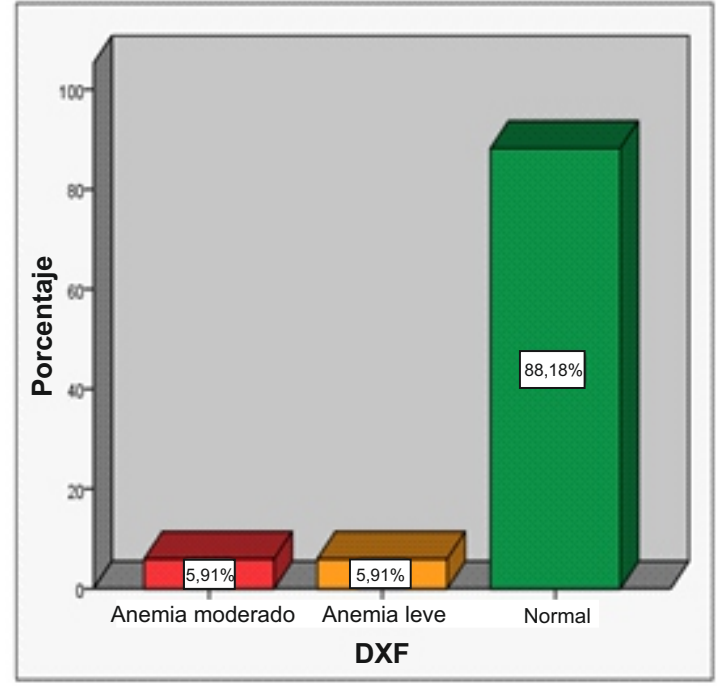

Fuente: Ficha de recolección de datos de historias clínicas aplicado por los investigadores.

En esta figura $\mathrm{N}^{0} 5$ se puede observar que del total de los niños en estudio que fueron 220, el diagnóstico final luego de la administración del sulfato ferroso fue de Normal un $88.18 \%$, seguido de anemia leve y anemia moderada, ambos con un $5.92 \%$.

Por lo cual esto indica que la mayoría de los niños que recibieron el sulfato ferroso resultaron con conseguir incrementar su hemoglobina, evidenciándose con los resultados de ya no tener anemia en su gran mayoría. 
TABLA $\mathbf{N}^{0}$ 4. Diagnóstico final de los niños con diagnóstico inicial de anemia después de la administración del sulfato ferroso

\begin{tabular}{|c|c|c|c|c|c|}
\hline & \multicolumn{5}{|c|}{ Diagnostico Final } \\
\hline & & $\begin{array}{l}\text { Anemia } \\
\text { hoderada }\end{array}$ & $\begin{array}{c}\text { Anemi } \\
\text { leve }\end{array}$ & a ${ }^{\text {Normal }}$ & I Total \\
\hline \multirow{2}{*}{$\begin{array}{c}\text { Diagnostico } \\
\text { Inicial }\end{array}$} & $\begin{array}{c}\text { Anemia } \\
\text { moderada }\end{array}$ & 13 & 13 & 73 & 99 \\
\hline & $\begin{array}{l}\text { Anemia } \\
\text { leve }\end{array}$ & 0 & 0 & 121 & 121 \\
\hline Total & & 13 & 13 & 194 & 220 \\
\hline
\end{tabular}

Fuente: Ficha de recolección de datos de historias clínicas aplicado por los investigadores.

La tabla $\mathrm{N}^{\circ} 4$ muestra la contingencia del diagnóstico inicial del tamizaje de hemoglobina de los niños y el diagnóstico final del control de hemoglobina, donde se observa que hay 121 (55\%) niños que tenían Anemia leve en el diagnóstico inicial pasaron a tener una hemoglobina de Normal en el diagnóstico final, como consecuencia de la administración del sulfato ferroso, hay $73(33,18 \%)$ niños que tenían Anemia moderada antes de administrarles sulfato ferroso y luego pasaron a tener un estado nutricional Normal, hay $13(5,9 \%)$ niños que tenían Anemia moderada y pasaron a tener Anemia leve, hay 13 (5,9\%) niños que se han mantenido con Anemia moderada antes y después de administrarles sulfato ferroso.

Estos resultados permiten aseverar la influencia de la administración del sulfato ferroso en los niños, ya que se ha incrementado significativamente la cantidad de hemoglobina en los niños del distrito de Huancayo.

TABLA $\mathbf{N}^{0} \mathbf{5}$. Diagnóstico final de hemoglobina y la influencia de la administración de sulfato ferroso en los niños de educación inicial

\begin{tabular}{ccccccc}
\hline \multicolumn{5}{c}{ Influencia del sulfato ferroso } \\
Malo Regular & Bueno Excelente Total \\
\hline & $\begin{array}{c}\text { Anemia } \\
\text { moderada }\end{array}$ & 4 & 5 & 2 & 13 \\
& $\begin{array}{c}\text { Anemia } \\
\text { leve }\end{array}$ & 1 & 5 & 4 & 3 & 13 \\
Normal & 2 & 2 & 25 & 165 & 194 \\
\hline & Total & 5 & 11 & 34 & 170 & 220
\end{tabular}

Fuente: Elaboración propia

Se observa, en la tabla $\mathrm{N}^{\circ} 5$ la contingencia del diagnóstico final de hemoglobina y la influencia de la administración del sulfato ferroso, donde se observa que hay 165 (75\%) niños que tienen hemoglobina Normal y una Excelente administración del sulfato ferroso, hay $25(11,36 \%)$ niños que tienen una hemoglobina Normal y una Buena administración del sulfato ferroso, hay $2(0,9 \%)$ niños que tienen hemoglobina normal y con una regular administración del sulfato ferroso, incluso hay $2(0,9 \%)$ niños más que tienen hemoglobina normal y una mala administración del sulfato ferroso. Estos resultados permiten aseverar que la influencia de la administración del sulfato ferroso en los niños con anemia en su gran mayoría sirve, ya que se ha incrementado significativamente la cantidad de hemoglobina en los niños del Distrito de 
Huancayo, a pesar que el tratamiento de sulfato ferroso no fue diario, sino de lunes a viernes, administrado con apoyo de las docentes del nivel inicial.

TABLA Nº. Prueba chi cuadrada de independencia de para la hipótesis de investigación

\begin{tabular}{lrrr}
\hline & & \multicolumn{2}{r}{$\begin{array}{r}\text { Significación } \\
\text { asintótica } \\
\text { (bilateral) }\end{array}$} \\
\hline $\begin{array}{l}\text { Chi-cuadrado de } \\
\text { Pearson }\end{array}$ & $85,477^{\text {a }}$ & 6 &, 000 \\
$\begin{array}{l}\text { Razón de } \\
\text { verosimilitud }\end{array}$ & 59,035 & 6 &, 000 \\
$\begin{array}{l}\text { Asociación lineal } \\
\text { por lineal }\end{array}$ & 67,765 & 1 &, 000 \\
N de casos válidos & 220 & & \\
\hline
\end{tabular}

a. 7 casillas $(58.3 \%)$ han esperado un recuento menor que 5. El recuento mínimo esperado es .30 .

La Tabla N ${ }^{\circ} 6$ muestra el valor de la Chi cuadrada calculada es $\mathrm{X}_{\mathrm{c}}{ }_{\mathrm{c}}=85,477$ y el $\mathrm{p}$ valor $(0,000)$ es menor al nivel de significación $(\alpha=0,050)$, por lo que se rechaza la hipótesis nula $\left(\mathrm{H}_{0}\right)$ y se acepta la hipótesis alterna $\left(\mathrm{H}_{1}\right)$ para un 95\% de nivel de confianza. Se utiliza la prueba Chi cuadrada de independencia. Esta tabla muestra las frecuencias observadas necesarias para realizar la prueba de la hipótesis.

\section{DISCUSIÓN}

Basándonos en la Teoría de Nola Pender en su Modelo de Promoción de la Salud, nos sirvió como investigadoras para identificar conceptos relevantes sobre las conductas de Promoción de la Salud, en nuestra investigación lo tomamos muy en cuenta en la actividad de haberse cumplido la administración del sulfato ferrosos en los niños con anemia, con lo cual se promueve el control en ellos para un buen tratamiento y mejora en su salud.

Es muy importante también mencionar que todos los estudios que se toman como antecedentes confirman que la administración del sulfato ferroso incrementa los niveles de hemoglobina, en beneficio del estado general del niño. La aceptación de la administración del sulfato ferroso respecto al cumplimiento de la dosis por parte de los niños fue en un $86,82 \%$ que tomaron la dosis completa y la administración del sulfato ferroso respecto al cumplimiento del periodo por parte de los niños fue en un $86,36 \%$. Lo cual refleja el resultado de que la influencia de la administración del sulfato ferroso a los niños de inicial del distrito de Huancayo esta categorizado en excelente en un porcentaje de $76.29 \%$, en bueno en un $15.81 \%$, en regular $5.15 \%$ y en malo en tan solo un $2.75 \%$.

De forma similar en un estudio de Moran A. y otros sobre Eficacia de la administración dos veces por semana de suplementos de sulfato ferroso para la reducción de la anemia en niños de 6 a 35 meses de edad, en comunidades rurales, realizado en Ancash encontró que la suplementación hecha por las Educadoras Comunales de Nutrición dos veces por semana es efectiva para la reducción de la anemia. 
Se observa en nuestro estudio en la tabla $\mathrm{N}^{\circ} 4$ muestra la contingencia del diagnóstico inicial del tamizaje de hemoglobina de los niños y el diagnóstico final del control de hemoglobina, donde se observa que hay 121 (55\%) niños que tenían Anemia leve en el diagnóstico inicial pasaron a tener una hemoglobina de Normal en el diagnóstico final, como consecuencia de la administración del sulfato ferroso, hay $73(33,18 \%)$ niños que tenían Anemia moderada antes de administrarles sulfato ferroso y luego pasaron a tener un estado nutricional Normal, hay 13 (5,9\%) niños que tenían Anemia moderada y pasaron a tener Anemia leve, hay 13 (5,9\%) niños que se han mantenido con Anemia moderada antes y después de administrarles sulfato ferroso. Estos resultados permiten aseverar la influencia de la administración del sulfato ferroso en los niños, ya que se ha incrementado significativamente la cantidad de hemoglobina en los niños del distrito de Huancayo.

Viendo la similitud de los resultados entre este estudio y el antecedente de otros estudios se afirma que la administración del Hierro en niños en forma de suplemento es eficaz, es decir influye para el tratamiento de la anemia ferropénica en los niños.

La contingencia del diagnóstico final de hemoglobina y la influencia de la administración del sulfato ferroso, donde se observa que hay 165 (75\%) niños que tienen hemoglobina Normal y una Excelente administración del sulfato ferroso, hay 25 (11,36\%) niños que tienen una hemoglobina Normal y una Buena administración del sulfato ferroso. Estos resultados permiten aseverar que la influencia de la administración del sulfato ferroso en los niños con anemia en su gran mayoría sirve, ya que se ha incrementado significativamente la cantidad de hemoglobina en los niños del Distrito de Huancayo.

El estudio muestra el valor de la Chi cuadrada calculada es $\mathrm{X}_{\mathrm{c}}{ }_{\mathrm{c}}=85,477$ y el $\mathrm{p}$ valor $(0,000)$ es menor al nivel de significación $(\alpha=0,050)$, por lo que se rechaza la hipótesis nula $\left(\mathrm{H}_{0}\right)$ y se acepta la hipótesis alterna $\left(\mathrm{H}_{1}\right)$ para un $95 \%$ de nivel de confianza.

Al rechazarse la validez de la hipótesis nula $\left(\mathrm{H}_{0}\right)$, se acepta la hipótesis alterna $\left(\mathrm{H}_{1}\right)$ : La administración del sulfato ferroso influye en el incremento de hemoglobina en niños con Anemia las instituciones de nivel inicial en el Distrito de Huancayo 2016, se concluye afirmando la hipótesis de investigación: La influencia de la administración del sulfato ferroso incrementa los niveles de hemoglobina en niños con Anemia las instituciones de nivel inicial en el Distrito de Huancayo 2016.

Por lo tanto, se hace necesario que, continuar con las actividades de tamizaje de hemoglobina para poder determinar en nuestros niños en edad pre escolar quienes tienen anemia y así poder darles oportunamente su tratamiento, mediante estrategias de salud, la Anemia estará latente en este grupo etario y con ello 
logrará perjudicar a nuestros niños en su crecimiento y desarrollo normal.

\section{CONCLUSIONES}

La administración del sulfato ferroso en niños con anemia es favorable, se puede observar en los niños de nivel inicial con anemia la influencia de la administración del sulfato ferroso en el diagnóstico final de hemoglobina, evidenciando que hay un porcentaje aceptable de niños que tuvieron una excelente administración del sulfato ferroso y resultaron en el control de hemoglobina con diagnóstico de Normal.

La administración del sulfato ferroso a los niños de nivel inicial con anemia del Distrito de Huancayo teniendo en cuenta el cumplimiento de la dosificación médica y el cumplimiento diario de la administración durante en tratamiento esta categorizado en excelente en su mayoría.

En la contingencia del diagnóstico inicial del tamizaje de hemoglobina de los niños y el diagnóstico final del control de hemoglobina después de la administración del sulfato ferroso se observa, que los niños que tenían Anemia leve en el diagnóstico inicial pasaron a tener una hemoglobina de Normal en su totalidad, los niños que tenían Anemia moderada en el diagnóstico inicial pasaron a tener una hemoglobina Normal en su mayoría, y estos niños que iniciaron con anemia moderada y no se recuperaron en su totalidad, la mitad después del tratamiento paso a tener anemia leve y la otra mitad continuo con moderada. Estos resultados permiten aseverar la influencia de la administración del sulfato ferroso en los niños con anemia, ya que se ha incrementado significativamente la cantidad de hemoglobina en estos niños.

En la contingencia del diagnóstico final de hemoglobina y la influencia de la administración del sulfato ferroso, se observa que hay una mayoría de niños que tienen hemoglobina Normal y una Excelente administración del sulfato ferroso, el resto de niños que tienen una hemoglobina Normal y con una Buena administración del sulfato ferroso, también hay 2 niños que tienen hemoglobina normal y con una regular administración del sulfato ferroso, incluso hay 2 niños más que tienen hemoglobina normal y una mala administración del sulfato ferroso. Estos resultados permiten aseverar que la influencia de la administración del sulfato ferroso en los niños con anemia en su gran mayoría sirve, ya que se ha incrementado significativamente la cantidad de hemoglobina en los niños del Distrito de Huancayo, a pesar que el tratamiento de sulfato ferroso no fue diario, sino de lunes a viernes, administrado con apoyo de las docentes del nivel inicial, de las instituciones educativas de donde eran los niños en estudio.

Este estudio se realizó el año 2016 dentro de las actividades del Programa Salud Escolar, la cual fue una estrategia de atención de salud para la población 
escolar que contribuyó a mejorar su calidad de vida, mediante el desarrollo de estilos de vida y entornos saludables, la detección de riesgos y daños en salud, así como su atención oportuna relacionada con el proceso de aprendizaje, en el marco de las políticas sectoriales MINSAMINEDU-MIDIS.

\section{RECOMENDACIONES}

Se recomienda a la DIRESA Junín, que realice un convenio con UGEL respecto a contar con las facilidades y el apoyo, para dar continuidad al trabajo del tamizaje de hemoglobina en los iniciales de la jurisdicción.

Se recomienda al personal de enfermería de los establecimientos de salud, el buscar estrategias para continuar realizando la actividad de tamizaje de hemoglobina a los niños en edad preescolar, su tratamiento si tuvieran anemia y su control para su futura alta.

Capacitación por parte del personal de salud a los profesores y padres de familia de niños de nivel inicial en temas de, loncheras saludables, consejerías nutricionales, para así sensibilizar a los docentes sobre la importancia de una alimentación saludable, que incluya además la temática de alimentación saludable en el programa escolar.

Desarrollar en el personal de enfermería estrategias de enseñanza- aprendizaje efectivas e interactivas como talleres, demostraciones de preparación de loncheras saludables dirigido a las madres de los niños de los iniciales, así permitan contribuir al desarrollo de hábitos alimenticios saludables en los niños en edad preescolar.

\section{REFERENCIAS BIBLIOGRÁFICAS}

OMS, Ginebra, Organización Mundial de la Salud, 2008.

The global prevalence of anaemia in 2011, World Health Organization.

Diario Perú 21 de fecha 17 de noviembre 2012 lima Perú.

Diario Perú 21 de fecha 02 de diciembre 2015 lima Perú.

Instituto Nacional de Estadística e Informática, "Perú Encuesta Demográfica y de Salud Familiar 2012 - Departamento de Junín”. Noviembre 2013.

Paola Estefanía Armijo Barragán influencia de la suplementación con hierro en el estado nutricional y niveles de hemoglobina de niños y niñas que asisten a los cibv, cantón publo viejo, provincia de los ríos. ecuador 2016 http://repositorio.unap.edu.pe/bits tream/handle/UNAP/2457/Paranc co_Rodriguez_Cyntia.pdf?sequen ce $=1 \&$ isAllowed $=y$

Ruiz, P. y Perez, A. Realizó en Chimborazo el 2013 la Tesis "Evaluación de la fase uno del programa de suplementación de 
cinco años, provincia de Chimborazo Ecuador-2013"

Cyntia Parancco Rodriguez. Realizó en Puno el 2014 la Tesis efecto de las prácticas de la suplementación del sulfato ferroso y consumo de hierro dietético en los niveles de hemoglobina en niños con anemia de 6 a 36 meses del puesto de salud Villa Socca, Acora. Disponible en: http://repositorio.unap.edu.pe/han dle/UNAP/2457

Moran A, Rodriguez D, Rojas M, Canchaya R. Realizaron en el 2012 la tesis eficacia de la administración dos veces por semana de suplementos de sulfato ferroso para la reducción de la anemia en niños de 6 a 35 meses de edad, en comunidades rurales de ancash, perú. Disponible en: h t $\mathrm{t}$ p s : / / g u z 1 o p editoras.com/web_des/med01/pe diatria/pld0619.pdf

Mengolé Amaya, P. Realizó la Tesis prevalencia de anemia y deficiencia de hierro en niños en etapa pre escolar y escolar en el centro educativo nacional 6020 del aa.hh. micaela bastidas, distrito de villa maría del triunfo, lima perú, 2013. Disponible en: http://www.acarrion.edu.pe/docu m e n tos / Prevale n c i a de\%20anemia-y-deficienciade\%20hierro-ni\%C3\%B1os-enetapa-escolar-\%20 escolar-uncentro $\% 20$ educativo.pdf
Becerril Grandez, Neisi. Realizó en el 2011 la Tesis eficacia del sulfato ferroso y multimicronutrientes en el incremento de la hemoglobina en los niños de 6 a 36 meses de edad, en los distritos de llamellin y san juan de rontoy en la provincia de antonio raimondi, ancash 2011. Disponible en: http://revistascientificas.upeu.ed u.pe/index.php/rc_salud/article/v iew/190

Centro de Atencion Farmaceutica (caf digemid)http://www.digemid.mi nsa.gob.pe/UpLoad/UpLoaded/P DF/Sulfato_Ferroso_Acido_F\% F3lico.pdf.

HIERRO https://www.webconsultas. com/dieta-y-nutricion/dietaequilibrada/micronutrientes/min erales/hierro-1833

\section{CORRESPONDENCIA:}

Lic. Mercedes Acosta Román

lic_mar_1@hotmail.com 